\title{
Failure of ETV in patients with the highest ETV success scores
}

\author{
Thomas J. Gianaris, MD, ${ }^{1}$ Ryan Nazar, MD, ${ }^{2}$ Emily Middlebrook, BS, ${ }^{3}$ David D. Gonda, MD, ${ }^{4}$ \\ Andrew Jea, MD, ${ }^{3}$ and Daniel H. Fulkerson, MD' \\ 'Department of Neurological Surgery, Division of Pediatric Neurosurgery, Goodman Campbell Brain and Spine, \\ Indiana University School of Medicine, Indianapolis, Indiana; ${ }^{2}$ Department of Neurosurgery, University of Louisville, \\ Kentucky; ${ }^{3}$ Department of Neurosurgery, Division of Pediatric Neurosurgery, Texas Children's Hospital, Baylor College of \\ Medicine, Houston, Texas; and “Division of Neurosurgery, University of California at San Diego, Rady Children's Hospital, \\ San Diego, California
}

OBJECTIVE Endoscopic third ventriculostomy (ETV) is a surgical alternative to placing a CSF shunt in certain patients with hydrocephalus. The ETV Success Score (ETVSS) is a reliable, simple method to estimate the success of the procedure by 6 months of postoperative follow-up. The highest score is 90 , estimating a $90 \%$ chance of the ETV effectively treating hydrocephalus without requiring a shunt. Treatment with ETV fails in certain patients, despite their being the theoretically best candidates for the procedure. In this study the authors attempted to identify factors that further predicted success in patients with the highest ETVSSs.

METHODS A retrospective review was performed of all patients treated with ETV at 3 institutions. Demographic, radiological, and clinical data were recorded. All patients by definition were older than 1 year, had obstructive hydrocephalus, and did not have a prior shunt. Failure of ETV was defined as the need for a shunt by 1 year. The ETV was considered a success if the patient did not require another surgery (either shunt placement or a repeat endoscopic procedure) by 1 year. A statistical analysis was performed to identify factors associated with success or failure.

RESULTS Fifty-nine patients met the entry criteria for the study. Eleven patients (18.6\%) required further surgery by 1 year. All of these patients received a shunt. The presenting symptom of lethargy statistically correlated with success ( $p$ $=0.0126$, odds ratio $[O R]=0.072)$. The preoperative radiological finding of transependymal flow $(p=0.0375,0 R 0.158)$ correlated with success. A postoperative larger maximum width of the third ventricle correlated with failure $(p=0.0265)$.

CONCLUSIONS The preoperative findings of lethargy and transependymal flow statistically correlated with success. This suggests that the best candidates for ETV are those with a relatively acute elevation of intracranial pressure. Cases without these findings may represent the failures in this highly selected group.

https://thejns.org/doi/abs/10.3171/2016.7.PEDS1655

KEY WORDS endoscopic third ventriculostomy; ETV Success Score; outcome; transependymal flow; hydrocephalus

$\mathrm{E}$ NDOSCOPIC third ventriculostomy (ETV) is an accepted alternative to CSF shunting for treatment of hydrocephalus. ETV has a high rate of success in selected patients. Kulkarni et al..$^{18}$ created the ETV Success Score (ETVSS; Table 1); this system provides a simple, reproducible method of estimating the chance of success of the procedure at 6 months. The ETVSS calculates a score based on 3 factors: patient age, cause of hydrocephalus, and the presence or absence of a previous shunt. The score approximates the odds that the ETV will effectively treat hydrocephalus without the need for shunt placement; the highest score possible is 90 , estimating a $90 \%$ chance of success. The highest rate of success is found in patients with obstructive hydrocephalus who have not had a prior shunt. In these patients, the ETVSS estimates an $80 \%$ chance of success in children from 1 to 10 years of age and a $90 \%$ chance in patients more than 10 years of age. However, ETV failure occurs in some patients with the highest

ABBREVIATIONS $\mathrm{Cl}=$ confidence interval; ETV = endoscopic third ventriculostomy; ETVSS = ETV Success Score; ICP = intracranial pressure; iNPH = idiopathic normal pressure hydrocephalus; OR = odds ratio. 
ETVSSs. We reviewed cases at 3 medical centers to evaluate if other factors may be considered to predict ETV success in patients with the highest ETVSSs.

\section{Methods \\ Study Population}

A retrospective review of all patients undergoing an ETV at Rady Children's Hospital (San Diego, California), Texas Children's Hospital (Houston, Texas), and Riley Hospital for Children (Indianapolis, Indiana) from 2010 to 2015 was performed. Patients with a preoperative ETVSS of 80 or 90 were identified. By definition, patients were older than 1 year, without a previous shunt, and with obstructive hydrocephalus. All patients included in the study had follow-up data of at least 1 year from the surgery. The study began after approval from each institution's medical review board.

\section{Data Collection}

Demographic information included the age, sex, and diagnosis of the patient. The clinical signs and symptoms were recorded. We considered the following symptoms to be suggestive of hydrocephalus: headache, nausea/vomiting, mental status changes, lethargy, visual abnormalities, developmental delay, gait instability, memory deficits, and incontinence. We recorded symptoms that were questionably related to hydrocephalus as "other." This category included 2 patients with tremor and single patients with spasticity, seizure, facial droop, delayed puberty, precocious puberty, and hemiplegia.

Preoperative radiological parameters included the maximum width of the third ventricle $(\mathrm{mm})$, maximum bicaudate distance $(\mathrm{mm})$, Evans index (ratio of the maximum width of the lateral ventricle frontal horns to the maximum transverse diameter of the inner table of the skull), and the presence or absence of transependymal flow. Postoperative radiological data also included an estimation of patency of the ETV. There was no standardized method for determining patency, although all patients were evaluated using MRI.

The clinical course of the patient was evaluated. "Failure" of the initial ETV was defined as the need for a shunt or further endoscopic surgery within 1 year. In this study, all patients whose ETV failed received a shunt; no patient underwent a repeat endoscopic procedure. The ETV was considered a "success" if the patient did not receive a shunt or further surgery by 1 year.

\section{Statistical Analysis}

Statistical analysis was performed using SAS software (version 9.4, SAS Inc.). Logistic regression models were used for the analysis of all variables of interest. The logistic regression model was chosen because the outcome of interest ("need for a shunt within 1 year") was considered to be binary. Interpretation of effects was recorded in terms of the odds ratio (OR).

For identifying significant effects, the stepwise model selection method was used for all variables related to demographic information, preoperative radiological parameters, and postoperative radiological parameters. In the
TABLE 1. Calculation of the ETVSS

\begin{tabular}{cllc}
\hline Score & \multicolumn{1}{c}{ Age } & \multicolumn{1}{c}{ Etiology } & $\begin{array}{c}\text { Previous } \\
\text { Shunt }\end{array}$ \\
\hline 0 & $<1$ mo & Postinfectious & Yes \\
\hline 10 & 1 mo to $<6$ mos & $\begin{array}{c}\text { Myelomeningocele, IVH, } \\
\text { nontectal brain tumor }\end{array}$ & No \\
20 & 6 mos to $<1$ yr & $\begin{array}{c}\text { Aqueductal stenosis, tectal } \\
\text { tumor, other }\end{array}$ & \\
\hline 30 & 1 yr to $<10$ yrs & \\
\hline 40 & $\geq 10$ yrs & & \\
\hline 50 & &
\end{tabular}

$\mathrm{IVH}=$ intraventricular hemorrhage.

Reproduced with permission from Kulkarni et al: J Neurosurg Pediatr 6:310-315, 2010.

* The ETVSS is calculated as age score + etiology score + previous shunt score.

stepwise procedure, the entry p value was set to 0.3 while the staying $\mathrm{p}$ value was 0.35 . Potential 2 -way interactions among the variables were also included in the stepwise model building process. A best model including only those selected variables was then fitted to obtain the associated $p$ values, OR estimates, and 95\% confidence intervals (CIs) for OR estimates.

For each of these nonselected variables in the stepwise procedure, a logistic regression model including only the variable of interest was also fitted to obtain its corresponding $\mathrm{p}$ value and OR estimate. Some of the variables had a very low event rate. To reduce the bias of estimates for such variables, Firth's method in logistic regression was applied.

\section{Results}

A total of 59 patients met all the entry criteria and were included in the study. All patients had an ETVSS of 80 or 90 . Thirty-two patients $(54.2 \%)$ had an ETVSS of 90 and $27(45.8 \%)$ had an ETVSS of 80 . There were 41 males $(69.5 \%)$ and 18 females (30.5\%). The demographic data are shown in Table 2. The most common diagnosis was aqueductal stenosis, which was given in 20 patients (33.9\%). The mean age of the patient population was 12.0 \pm 11.7 years (median 10 years). The estimated duration of symptoms prior to surgery was $8.4 \pm 15.3$ months. In 11 patients (18.6\%) the ETVs were considered failures. All of these patients had a shunt placed within 1 year; there were no endoscopic revisions and no deaths.

The presenting symptoms are shown in Table 3. The most common symptom was headache, appearing in 39 patients (66.1\%). Three symptoms (mental status changes, memory problems, and incontinence) occurred rarely enough that the ORs were not estimable using the ordinary maximum likelihood method (indicated in Table 3). The duration of symptoms did not statistically correlate with success. The symptom of lethargy was statistically significantly associated with success $(\mathrm{p}=0.0126, \mathrm{OR} 0.072)$. The category of "other" was also statistically correlated with success $(p=0.022)$. We did not draw conclusions from this category, given the heterogeneous and varied symptoms that may not have been related to hydrocephalus.

Physical examination findings are demonstrated in Ta- 
TABLE 2. Demographics of the study group $(n=59)$

\begin{tabular}{lc}
\hline \multicolumn{1}{c}{ Variable } & Value (\%) \\
\hline Sex & \\
Male & $41(69.5)$ \\
Female & $18(30.5)$ \\
\hline Age (yrs) & \\
Mean \pm SD & $12.0 \pm 11.7$ \\
Median & 10 \\
\hline Diagnosis & \\
Aqueductal stenosis & $20(33.9)$ \\
Tectal glioma & $13(22.0)$ \\
Pineal tumor & $17(28.8)$ \\
Other & $9(15.3)$ \\
\hline ETVSS & \\
90 & $32(54.2)$ \\
80 & $27(45.8)$ \\
\hline Failure (required shunt by $1 \mathrm{yr})$ & $11(18.6)$ \\
\hline
\end{tabular}

ble 4 . The most common sign was papilledema, occurring in 13 patients $(22.0 \%)$. There were no statistically significant correlations with outcome.

Both the pre- and postoperative MRI findings are shown in Table 5. The presence of preoperative transependymal flow was statistically correlated with success ( $\mathrm{p}=$ 0.0375 , OR 0.158). Postoperatively, a larger width of the third ventricle correlated with an increased risk of failure ( $\mathrm{p}=0.0265$, OR 1.128). This width was stepwise based on a unit of $1 \mathrm{~mm}$. For every $1 \mathrm{~mm}$ increase in size of the third ventricle, the odds of requiring a shunt were 1.128 greater $(12.8 \%)$.

\section{Illustrative Cases \\ Case 1}

A 10-year-old otherwise healthy child presented with 2 weeks of progressive, debilitating headaches with nausea and vomiting. He had difficulty walking, florid papilledema, ataxia, and lethargy.

His initial MRI showed dilated ventricles with transependymal flow (Fig. 1A). The obstructive hydrocephalus was caused by a mass in the pineal region (Fig. 1B). The patient underwent an ETV with tumor biopsy. His ETVSS was 80 (age $\geq 10$ years [50], nontectal brain tumor [20], and no previous shunt [10]). His symptoms completely resolved after surgery.

The pathological diagnosis was teratoma with both mature and immature elements. The patient initially underwent a treatment regimen of chemo- and radiation therapy. However, his tumor continued to expand. The patient then underwent subtotal open resection. The tumor has subsequently returned. However, the patient's symptoms and papilledema completely resolved after the ETV. He has not required any further treatment for hydrocephalus. An MR image obtained 5 years after the original surgery shows decompressed ventricles without transependymal flow (Fig. 1C). The aqueduct has never been radiologically open, so we consider the ETV to be the sole treatment for the hydrocephalus.
TABLE 3. Symptoms related to outcome

\begin{tabular}{lcccc}
\hline \multicolumn{1}{c}{ Symptom } & Value (\%) & OR & $95 \% \mathrm{Cl}$ & $\mathrm{p} \mathrm{Value}$ \\
\hline Headache & $39(66.1)$ & 1.833 & $0.483-6.964$ & 0.3734 \\
\hline Nausea/vomiting & $24(40.7)$ & 0.5 & $0.133-1.877$ & 0.3044 \\
\hline Mental status changes* & $1(1.7)$ & 0.72 & $0.008-69.081$ & 0.8879 \\
\hline Lethargy $^{*}$ & $5(8.5)$ & 0.072 & $0.009-0.568$ & $\mathbf{0 . 0 1 2 6}$ \\
\hline Vision problems & $18(30.5)$ & 1.212 & $0.281-5.222$ & 0.7963 \\
\hline Developmental delay & $5(8.5)$ & 0.909 & $0.091-9.034$ & 0.9352 \\
\hline Gait instability & $13(22.0)$ & 0.702 & $0.157-3.14$ & 0.6431 \\
\hline Memory problems* & $1(1.7)$ & 0.073 & $<0.001-7.002$ & 0.2608 \\
\hline Incontinence* & $2(3.4)$ & 1.237 & $0.028-53.97$ & 0.9121 \\
\hline Other & $8(13.6)$ & 0.108 & $0.016-0.726$ & 0.022 \\
\hline
\end{tabular}

Boldface type indicates statistical significance.

* Because the events for metal status changes, memory problems, and incontinence are very rare in the data, the corresponding ORs are not estimable using the ordinary maximum likelihood method. Their estimated ORs, 95\% Cls, and $p$ values are obtained through the penalized maximum likelihood approach.

\section{Case 2}

A 16-year-old boy presented with increasing headaches for 2 years. His visual acuity declined over the same time period. Physical examination demonstrated a very slow gait with toe walking. His head size was greater than 2 standard deviations from the mean. He attended special education classes and was diagnosed with "developmental delay" since infancy.

MRI showed grossly dilated lateral and third ventricles without transependymal flow (Fig. 2A). Sagittal imaging demonstrated tectal thickening with aqueductal stenosis and a relatively normal-sized fourth ventricle. The patient had an ETVSS of 90, and he underwent a technically successful ETV.

The patient's gait and headaches minimally improved. He did not change cognitively. His symptoms returned after a few months. Postoperative MRI demonstrated stable large ventricles. Sagittal T2-weighted MRI demonstrated a flow artifact through the ETV (Fig. 2B) per our interpretation, although the image is degraded due to his dental hardware. We believed that the ETV was patent, but that the patient was symptomatic from ventriculomegaly. A shunt was placed within 1 year of his ETV. The patient had minimal improvement in his gait after shunt placement.

TABLE 4. Physical examination findings related to outcome

\begin{tabular}{lcccl}
\hline Examination Finding & Value (\%) & OR & $95 \% \mathrm{Cl}$ & p Value \\
\hline Macrocephaly & $11(18.6)$ & 1.038 & $0.191-5.654$ & 0.9654 \\
\hline Papilledema & $13(22.0)$ & 3.332 & $0.386-28.794$ & 0.274 \\
\hline $\begin{array}{l}\text { Cranial nerve palsy/ } \\
\quad \text { Parinaud's }\end{array}$ & $12(20.3)$ & 2.972 & $0.342-25.847$ & 0.3236 \\
\hline Bradycardia & $3(5.1)$ & 0.435 & $0.036-5.275$ & 0.5131 \\
\hline Motor deficits/ataxia* $^{*}$ & $6(10.1)$ & 3.519 & $0.147-84.307$ & 0.4375 \\
\hline
\end{tabular}

* As in Table 3, the OR for motor deficits/ataxia was obtained through the penalized maximum likelihood approach. 
TABLE 5. Imaging parameters related to outcome

\begin{tabular}{lcccc}
\hline \multicolumn{1}{c}{ Parameter } & Measurement & OR & $95 \% \mathrm{Cl}$ & $\mathrm{p} \mathrm{Value}$ \\
\hline Preop MRI $(\mathrm{n}=58)$ & & & & 0.3293 \\
\hline Mean max width of 3rd ventricle \pm SD $(\mathrm{mm})$ & $14.8 \pm 6.4$ & 1.05 & $0.952-1.157$ & 0.1453 \\
\hline Mean max bicaudate distance \pm SD $(\mathrm{mm})$ & $34.2 \pm 12.3$ & 1.037 & $0.987-1.09$ & 0.0804 \\
\hline Mean Evans index \pm SD & $0.40 \pm 0.11$ & 155.723 & 0.542 to $>999.99$ & 0.0375 \\
\hline Transependymal flow (\%) & $26(44.8)$ & 0.158 & $0.028-0.899$ & 0.0265 \\
\hline Postop MRI $(\mathrm{n}=57)$ & & & & 0.0617 \\
\hline Mean max width of 3rd ventricle \pm SD $(\mathrm{mm})$ & $11.5 \pm 6.4$ & 1.128 & $1.014-1.255$ & 0.0465 \\
\hline Mean max bicaudate distance \pm SD $(\mathrm{mm})$ & $27.8 \pm 12.1$ & 1.052 & $0.997-1.11$ & 1.12 to $>999.99$ \\
\hline Mean Evans index \pm SD & $0.36 \pm 0.09$ & $>999.99$ & $0.009-4.471$ & 0.3115 \\
\hline Transependymal flow $(\%)$ & $8(14.0)$ & 0.202 & & \\
\hline
\end{tabular}

Boldface type indicates statistical significance.

\section{Case 3}

A 43-year-old man was evaluated for progressive headaches, mild dysconjugate gaze, and memory difficulties. He stated that he had had headaches since childhood, but they were worsening in intensity, duration, and frequency over the previous 6 months. His symptoms were interfering with his job as an engineer. He had a normal neurological examination without papilledema. He had an occipitofrontal circumference measurement of $59 \mathrm{~cm}$. He tried a number of medical headache regimens without success.

MRI demonstrated enlarged lateral and third ventricles, with a relatively spared fourth ventricle (Fig. 3A and B). We believed that the radiological appearance, clinical presentation, and macrocephaly suggested longstanding obstructive hydrocephalus. The patient underwent a technically successful ETV. He had an ETVSS of 90.

The patient had minimal to no change in his headaches or memory after surgery. MRI 9 months after surgery demonstrated a flow artifact through the ETV (Fig. 3C) with stable ventricle size. The patient eventually underwent shunt placement, but his symptoms did not improve afterward.

\section{Discussion}

The complications of CSF shunting are well known; complications include infection, operative morbidity, overdrainage, and a rate of malfunction of as much as $50 \%$ by 2 years. ${ }^{2,10}$ In the US alone, the cost of CSF shunting in patients and managing their complications approaches \$2 billion annually. ${ }^{26}$ ETV is a desirable alternative to shunt placement in certain patients. In selected patients, ETV provides a high rate of success and similar quality of life compared with shunting. ${ }^{8,13,20}$ ETV offers the advantage of effectively treating hydrocephalus without the need for a shunt.

There are potential complications with performing an ETV. ${ }^{5}$ Minor complications include CSF leak, infection, subdural hygromas, and hematomas. Major complications include endocrine abnormalities, injury to neural structures (oculomotor nerve, fornix, thalamus, hypothalamus), and possibly fatal injury to the basilar artery or basilar perforators. The complication rate is generally accepted to be related to the experience of the surgeon..$^{25}$ The reported complication rate ranges from $0 \%$ to $31 \%$, with an overall estimation of approximately $8.5 \%,{ }^{1,4,7,12,16}$ The risk of basilar artery injury is less than $0.2 \%$ in experienced hands, although the risk of abandoning the procedure because of intraoperative hemorrhage is approximately $1 \%{ }^{1,3,5}$ Given the rare but definite risks of the procedure, surgeons must properly select patients who have a realistic chance of success.
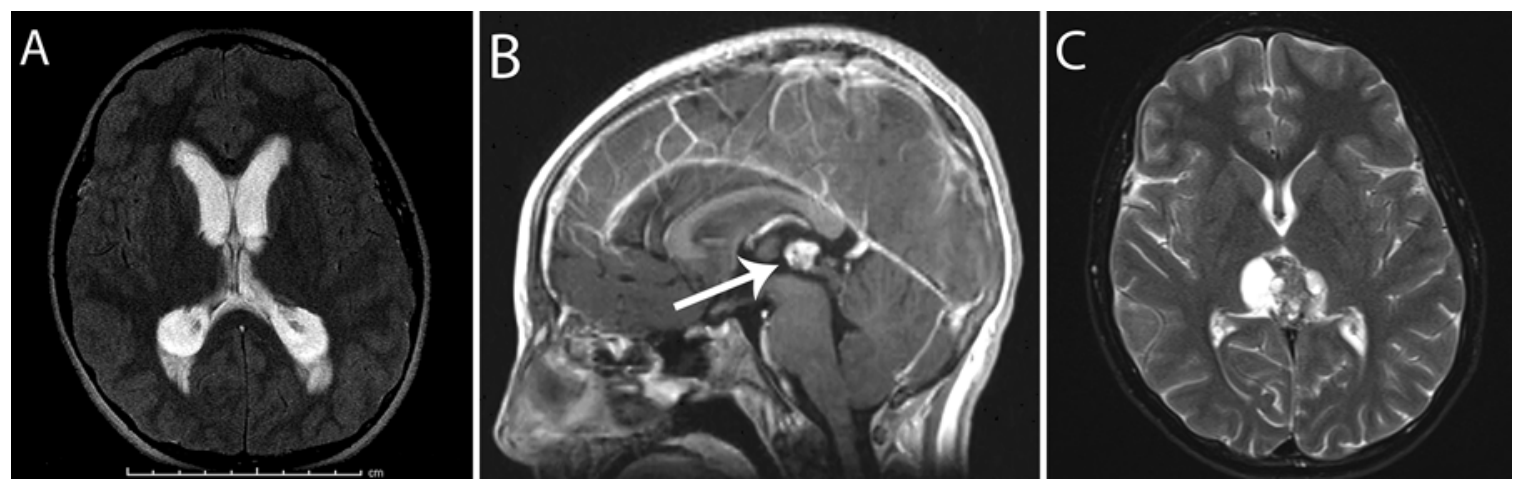

FIG. 1. Case 1. A: Axial FLAIR MR image demonstrates dilated ventricles with transependymal flow. B: Sagittal T1-weighted MRI with contrast demonstrates an enhancing pineal region mass (arrow). C: Axial T2-weighted MRI 5 years after initial treatment shows residual tumor, but resolution of the hydrocephalus with decompressed ventricles and resolution of the transependymal flow. 

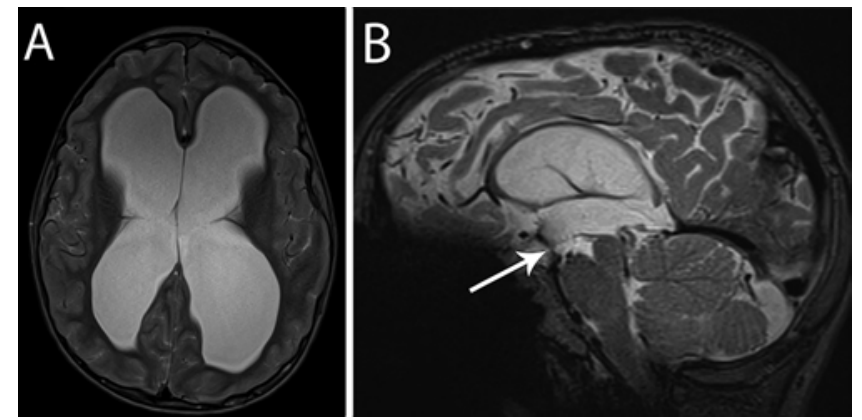

FIG. 2. Case 2. A: Axial T2-weighted MRI demonstrates enlarged ventricles without transependymal flow. B: Ten months after surgery, the sagittal T2-weighted MRI shows a flow artifact (arrow) suggesting flow through the ETV.

The ETVSS was developed to predict the success rate of an ETV by 6 months. ${ }^{19}$ This scale is simple, intuitive, and has been validated both by the original authors and by other centers. ${ }^{11,18,21,22,28,30}$ Kulkarni et al. based the score on 3 factors: age of the patient, origin of hydrocephalus, and the presence/absence of a previous shunt. The highest score is 90 , predicting a $90 \%$ success rate at 6 months. Prior authors have explored methods to increase the chance of success in patients with low ETVSS scores. ${ }^{27}$ However, published data are scarce regarding failure in patients who are the theoretically best candidates.

In this study, we evaluated 59 patients with an ETVSS of 80 or 90 . We defined ETV failure as the requirement of shunt placement by 1 year. Eleven patients (18.6\%) required a shunt, leaving a success rate of $81.4 \%$. This is slightly lower than the estimate of $85.4 \%$, derived by averaging all the patients' scores.

The ideal outcome is demonstrated in Case 1. In this patient, ETV led to resolution of the hydrocephalus with 5 years of follow-up data, despite multiple treatments for a complex tumor. This patient showed objective radiological improvement with a decreased ventricle size and resolution of transependymal flow. Clinically, his papilledema resolved and he had no further symptoms of hydrocephalus. By any definition, the ETV in this case was a success.

However, the definition of success or failure in ETV may be ambiguous. Previous authors have defined success as the absence of further CSF diversionary procedures, the absence of death from hydrocephalus, and improvement in clinical symptoms. ${ }^{9,15,23}$ The absence of death is an objective measure. However, improvement in clinical symptoms may be subjective, as shown in Cases 2 and 3. Both of these patients underwent technically successful procedures, but they did not clinically improve. Both later underwent shunt placement, and thus their cases qualified as treatment failures in this study.

We statistically evaluated multiple clinical and radiological findings in an attempt to identify factors that may further predict outcome. There was no significant difference in outcome based on diagnosis in this restricted population. There was a statistically significant association of success with the symptoms of lethargy $(p=0.0126$, OR 0.072). The statistical interpretation of this ratio is that the odds of a patient presenting with lethargy receiving a shunt by 1 year are 0.07 times the odds of a patient without lethargy. Table 4 shows the presenting signs of the study group. None of the signs were statistically correlated with the outcome, including papilledema.

Multiple radiological factors were studied. The preoperative presence of transependymal flow statistically correlated with success $(\mathrm{p}=0.0375$, OR 0.158$)$. The preoperative ventricular size and Evans ratio did not predict outcome. Postoperatively, there was a correlation between outcome and maximum third ventricle width $(\mathrm{p}=0.0265)$. A larger postoperative width correlated with an increased risk of failure (OR 1.128). Thus, for every $1 \mathrm{~mm}$ increase in size of the third ventricle, the odds of requiring a shunt were 1.128 greater $(12.8 \%)$.

The average duration of symptoms was 8.3 months, but this did not statistically correlate with outcome. There was significant subjectivity in recording the duration of symptoms. For example, the patient in Case $3 \mathrm{had}$ a $59-\mathrm{cm}$ head circumference, suggesting some measure of hydrocephalus occurring prior to fusion of the cranial sutures. He reported headaches since childhood. However, his duration of symptoms was recorded as 6 months, as this was the time of worsening symptoms.

Patients with ventriculomegaly may have varying degrees of elevation of intracranial pressure (ICP). Our
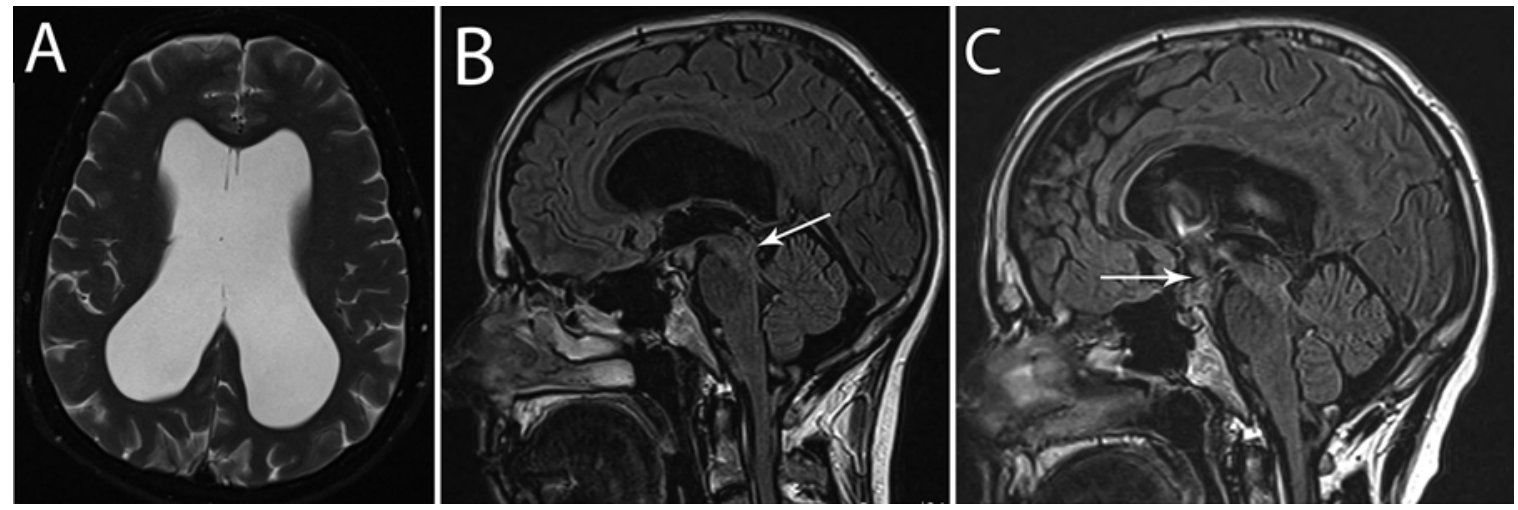

FIG. 3. Case 3. A: Axial T2-weighted MRI demonstrates enlarged ventricles without transependymal flow. B: The sagittal FLAIR MRI shows compression of the cerebral aqueduct (arrow). C: The sagittal FLAIR MRI 9 months after ETV demonstrated an artifact (arrow) suggesting flow through the stoma. 
data suggest that patients with elevated ICP are the best candidates for ETV. The symptom of lethargy suggests a relative acute rise in ICP. In these patients, we speculate that the surrounding brain parenchyma retains its normal turgor and compliance. Elevated intraventricular pressure with normal brain parenchymal compliance may cause transependymal flow, which was statistically correlated with success in our study. As the brain parenchyma retains its normal elastic properties, it is able to "push back" against the rise in intraventricular pressure. Once an ETV is performed, the brain parenchyma resistance forces fluid through the stoma and shrinks the ventricles. A smaller size of the third ventricle after surgery was statistically correlated with success $(p=0.0265)$. We noted that a larger postoperative third ventricle width correlates with failure; thus, a smaller third ventricle size correlates with success. The patient in Case 1 demonstrates both clinical resolution of symptoms and reduction in ventricle size.

The Case 1 patient contrasts with the patients in Cases 2 and 3. These patients had ventriculomegaly but a lower intraventricular pressure and possibly an altered brain parenchymal compliance. This may occur in patients with compensated hydrocephalus where the ventricles enlarge slowly over an extended period of time. The authors speculate that these patients have abnormal brain compliance in which the parenchyma has lost the elasticity to push back against the enlarged ventricles. Therefore, there is no pressure gradient encouraging sufficient flow through the ETV.

The patient in Case 2 had chronically enlarged ventricles. He had enlargement of the lateral and third ventricle, with a relatively spared fourth ventricle. His ventricles did not substantially shrink after a technically successful ETV. We speculate that his symptoms were analogous to idiopathic normal pressure hydrocephalus (iNPH) and related to chronic ventriculomegaly as opposed to elevated ICP. While surgeons have attempted ETV for elderly patients with iNPH, ${ }^{14}$ the published results have not convincingly demonstrated efficacy. ${ }^{24,29}$ The clinical triad of findings of iNPH (dementia, gait disturbance, and incontinence) are well known, but the pathophysiology is not understood. There is speculation that symptoms are related to stretching of the subcortical motor and dopaminergic pathways and the frontostriatal pathways. ${ }^{6,17}$ Some patients respond to shunt placement, presumably from active decrease of the ventricle size. The patient in Case 2 had a technically successful ETV but did not improve. He improved minimally with shunt placement. While the ETVSS does not differentiate this patient from the child in Case 1, we would suggest that his hydrocephalus is completely different.

The adult patient in Case 3 was included to highlight the challenge of treating patients with vague, nonspecific symptoms. The patient had radiological evidence of enlargement of his lateral and third ventricle. His condition was clearly longstanding, demonstrated by his macrocephaly. Our initial estimation was that his symptoms were unlikely to improve with surgery. We maximized medical management, but he subjectively reported that his symptoms were hindering his ability to perform his job and were a significant detriment to his quality of life. One may justifiably debate the diagnosis of "hydrocephalus" in this patient. However, if the clinical judgment is that the patient has hydrocephalus, the ETVSS does not discriminate between this patient and the patient in Case 1. His ETVSS predicted a $90 \%$ chance of success. He did not improve after the procedure (or after shunt placement). Cases 2 and 3 demonstrate the inherent complexities in the etiology and treatment of hydrocephalus.

\section{Limitations of the Study}

While all surgeons in the study were experienced in the procedure, there was no standardized surgical protocol. As mentioned, there was significant subjectivity in the decision to place a shunt in certain patients. This is illustrated in Case 3. This decision was left to the judgment of the surgeon and there was no standardized protocol.

The main limitation of this study is the relatively small number of patients. We were highly selective in our inclusion criteria as we attempted to focus on a very specific group of patients. However, this restrictive inclusion limits the overall number of patients. Thus, the statistical analysis and the conclusions should be considered suggestions. Any further evaluation of the ETVSS in these patients will require a more national evaluation.

\section{Conclusions}

The ETVSS is a pragmatic, simple, and useful tool to predict the outcome of ETV. However, there are treatment failures in patients with the highest possible scores. In this cohort of patients with ETVSSs of 80 or 90, we identified factors that further predict success. In this small but focused study, our data suggest that the highest chance of success occurs in patients with elevated ICP, demonstrated by lethargy and transependymal flow. This is in contrast to patients with ventriculomegaly but a lower or even normal ICP. However, this difference is not reflected by differences in the ETVSS. Postoperatively, success was correlated with smaller ventricles, specifically the third ventricle. A larger postoperative third ventricle width was related to failure. We suggest that these factors be considered when counseling patients regarding the efficacy of ETV.

\section{Acknowledgments}

We thank Yuanfang Xu and George Eckert of the Indiana University Department of Biostatistics for the statistical analysis. We also thank the Goodman Campbell Foundation for financial support.

\section{References}

1. Amini A, Schmidt RH: Endoscopic third ventriculostomy in a series of 36 adult patients. Neurosurg Focus 19(6):E9, 2005

2. Aschoff A, Kremer P, Hashemi B, Kunze S: The scientific history of hydrocephalus and its treatment. Neurosurg Rev 22:67-95, 1999

3. Baykan N, Isbir O, Gerçek A, Dağçnar A, Ozek MM: Ten years of experience with pediatric neuroendoscopic third ventriculostomy: features and perioperative complications of 210 cases. J Neurosurg Anesthesiol 17:33-37, 2005

4. Bouras T, Sgouros S: Complications of endoscopic third ventriculostomy. J Neurosurg Pediatr 7:643-649, 2011

5. Bouras T, Sgouros S: Complications of endoscopic third ven- 
triculostomy. World Neurosurg 79 (2 Suppl):S22.e9-S22. e12, 2013

6. Bradley WG: Normal pressure hydrocephalus: new concepts on etiology and diagnosis. AJNR Am J Neuroradiol 21:1586-1590, 2000

7. Brockmeyer D, Abtin K, Carey L, Walker ML: Endoscopic third ventriculostomy: an outcome analysis. Pediatr Neurosurg 28:236-240, 1998

8. Chhun V, Sacko O, Boetto S, Roux FE: Third ventriculocisternostomy for shunt failure. World Neurosurg 83:970-975, 2015

9. Drake JM: Endoscopic third ventriculostomy in pediatric patients: the Canadian experience. Neurosurgery 60:881-886, 2007

10. Drake JM, Kestle JR, Milner R, Cinalli G, Boop F, Piatt J Jr, et al: Randomized trial of cerebrospinal fluid shunt valve design in pediatric hydrocephalus. Neurosurgery 43:294-305, 1998

11. Durnford AJ, Kirkham FJ, Mathad N, Sparrow OC: Endoscopic third ventriculostomy in the treatment of childhood hydrocephalus: validation of a success score that predicts long-term outcome. J Neurosurg Pediatr 8:489-493, 2011

12. Dusick JR, McArthur DL, Bergsneider M: Success and complication rates of endoscopic third ventriculostomy for adult hydrocephalus: a series of 108 patients. Surg Neurol 69:5-15, 2008

13. Furlanetti LL, Santos MV, de Oliveira RS: The success of endoscopic third ventriculostomy in children: analysis of prognostic factors. Pediatr Neurosurg 48:352-359, 2012

14. Gangemi M, Maiuri F, Naddeo M, Godano U, Mascari C, Broggi G, et al: Endoscopic third ventriculostomy in idiopathic normal pressure hydrocephalus: an Italian multicenter study. Neurosurgery 63:62-69, 2008

15. Grand W, Leonardo J, Chamczuk AJ, Korus AJ: Endoscopic third ventriculostomy in 250 adults with hydrocephalus: patient selection, outcomes, and complications. Neurosurgery 78:109-119, 2016

16. Hader WJ, Walker RL, Myles ST, Hamilton M: Complications of endoscopic third ventriculostomy in previously shunted patients. Neurosurgery 63 (1 Suppl 1):ONS168ONS175, 2008

17. Knutsson E, Lying-Tunell U: Gait apraxia in normal-pressure hydrocephalus: patterns of movement and muscle activation. Neurology 35:155-160, 1985

18. Kulkarni AV, Drake JM, Kestle JR, Mallucci CL, Sgouros S, Constantini S: Predicting who will benefit from endoscopic third ventriculostomy compared with shunt insertion in childhood hydrocephalus using the ETV Success Score. J Neurosurg Pediatr 6:310-315, 2010

19. Kulkarni AV, Drake JM, Mallucci CL, Sgouros S, Roth J, Constantini S, et al: Endoscopic third ventriculostomy in the treatment of childhood hydrocephalus. J Pediatr 155:254259, 259.e1, 2009

20. Kulkarni AV, Shams I, Cochrane DD, McNeely PD: Quality of life after endoscopic third ventriculostomy and cerebrospinal fluid shunting: an adjusted multivariable analysis in a large cohort. J Neurosurg Pediatr 6:11-16, 2010

21. Labidi M, Lavoie P, Lapointe G, Obaid S, Weil AG, Bojanowski MW, et al: Predicting success of endoscopic third ventriculostomy: validation of the ETV Success Score in a mixed population of adult and pediatric patients. J Neurosurg 123:1447-1455, 2015
22. Naftel RP, Reed GT, Kulkarni AV, Wellons JC: Evaluating the Children's Hospital of Alabama endoscopic third ventriculostomy experience using the Endoscopic Third Ventriculostomy Success Score: an external validation study. J Neurosurg Pediatr 8:494-501, 2011

23. Sacko O, Boetto S, Lauwers-Cances V, Dupuy M, Roux FE: Endoscopic third ventriculostomy: outcome analysis in 368 procedures. J Neurosurg Pediatr 5:68-74, 2010

24. Sankey EW, Jusué-Torres I, Elder BD, Goodwin CR, Batra S, Hoffberger J, et al: Functional gait outcomes for idiopathic normal pressure hydrocephalus after primary endoscopic third ventriculostomy. J Clin Neurosci 22:1303-1308, 2015

25. Santamarta D, Díaz Alvarez A, Gonçalves JM, Hernández J: Outcome of endoscopic third ventriculostomy. Results from an unselected series with noncommunicating hydrocephalus. Acta Neurochir (Wien) 147:377-382, 2005

26. Simon TD, Riva-Cambrin J, Srivastava R, Bratton SL, Dean JM, Kestle JR: Hospital care for children with hydrocephalus in the United States: utilization, charges, comorbidities, and deaths. J Neurosurg Pediatr 1:131-137, 2008

27. Stone SS, Warf BC: Combined endoscopic third ventriculostomy and choroid plexus cauterization as primary treatment for infant hydrocephalus: a prospective North American series. J Neurosurg Pediatr 14:439-446, 2014

28. Tewuerbati S, Maimaitili M, Zhu G, Du G, Liu B, Sailike D, et al: Timing of endoscopic third ventriculostomy in pediatric patients with congenital obstructive hydrocephalus: assessment of neurodevelopmental outcome and short-term operative success rate. J Clin Neurosci 22:1292-1297, 2015

29. Tudor KI, Tudor M, McCleery J, Car J: Endoscopic third ventriculostomy (ETV) for idiopathic normal pressure hydrocephalus (iNPH). Cochrane Database Syst Rev 7:CD010033, 2015

30. Warf BC, Mugamba J, Kulkarni AV: Endoscopic third ventriculostomy in the treatment of childhood hydrocephalus in Uganda: report of a scoring system that predicts success. J Neurosurg Pediatr 5:143-148, 2010

\section{Disclosures}

The authors report no conflict of interest concerning the materials or methods used in this study or the findings specified in this paper.

\section{Author Contributions}

Conception and design: Fulkerson, Gianaris, Nazar, Jea. Acquisition of data: all authors. Analysis and interpretation of data: Fulkerson, Jea. Drafting the article: Fulkerson, Gianaris, Nazar. Critically revising the article: Fulkerson. Approved the final version of the manuscript on behalf of all authors: Fulkerson. Administrative/technical/material support: Fulkerson. Study supervision: Fulkerson.

\section{Correspondence}

Daniel H. Fulkerson, Indiana University School of Medicine/ Goodman Campbell Brain and Spine, Riley Hospital for Children, 702 Barnhill Dr. \#1134, Indianapolis, IN 46202-5200. email: dfulkers@iupui.edu. 